\title{
Visual Information Retrieval with the SuperTable + Scatterplot
}

\author{
Peter Klein $^{1}$, Frank Müller ${ }^{1}$, Harald Reiterer ${ }^{1}$, and Maximilian Eibl ${ }^{2}$ \\ ${ }^{1}$ University of Konstanz, Department of Computer \& Information Science, \\ Universitätsstr. 10, 78457 Konstanz, Germany \\ \{peter.klein, frank.mueller, harald.reiterer\}@uni-konstanz.de \\ 2 IZ Social Science Information Centre, Schiffbauerdamm 19, 10117 Berlin, Germany \\ eibl@berlin.iz-soz.de
}

\begin{abstract}
We present a new visualization approach for metadata combining different visualizations into a so-called SuperTable accompanied by a Scatterplot. The goal is to improve user experience during the information seeking process. Our new visualizations are based on our experiences developing a visual information retrieval system called INSYDER to supply small and medium size enterprises with business information from the Internet. Based on extensive user tests the original visualizations have been redesigned in two different design variants. Instead of offering multiple visualizations to choose from the SuperTable + Scatterplot combines them in a new way. Therefore, the user has the feeling that he is working with one single visualization in different states. Further the SuperTable solves a problem which seemed to be immanent to visualizations in document retrieval: the change of modalities.
\end{abstract}

\section{Introduction}

Conventional document retrieval systems return long lists of ranked documents that users are forced to sift through to find relevant documents. The majority of today's web search engines follow this paradigm. Surveys have shown that users have problems with the current paradigm of information retrieval systems for Web search simply presenting a long list of results. These long lists of results are not very intuitive for finding the most relevant documents in the result set. These empirical findings motivated us to develop a new type of user interface for Web retrieval that supports the user in the information seeking process by providing special visualizations in addition to the traditional result list. Systems combining the functionality of retrieval systems with the possibilities of information visualization systems [2] are called visual information retrieval systems.

This paper presents our main design ideas developing such a visual information retrieval system. Section 2 summarizes our experiences developing and evaluating the first version of our visual information retrieval system called INSYDER. Based on a comprehensive empirical user test with 40 users we have made a redesign of the original INSYDER visualizations. Section 3 presents in detail the redesigned visualizations, the so-called SuperTable supplemented by a Scatterplot. We are currently developing two different versions of the SuperTable using different strategies showing the granularity of details (levels of details versus a smooth change of granularity approach). Section 4 discusses related work that has influenced our visualizations. Conclusions and an outlook are given in section 5 .

\section{A Visual Information Retrieval System for the Web}

The first implementation of the INSYDER ${ }^{1}$ system includes five visualizations for the presentation of search results $[8,11,12]$ : a traditional list (mainly for evaluation purposes), a ResultTable, a ScatterPlot, a BarGraph, and a SegmentView with two modes: TileBars and StackedColumns. The primary intention for the use of different visualizations was to present additional information (metadata) about the retrieved documents to the user in a way that is intuitive, may be quickly interpreted, and can scale to large document sets.

An extensive evaluation done with 40 users [9] has been focused on the different visualizations used to present the search results in the result phase of the search process. The primary goal of this summative evaluation was to determine the usability of the visualizations. A second goal was to detect problems with the visualizations used in the INSYDER system, and to collect suggestions for improvements. The usability evaluation part of the study was focused on the added value of the visualizations (ScatterPlot, BarGraph, TileBar, StackedColumn) in terms of their effectiveness (accuracy and completeness with which users achieve task goals), efficiency (the task time users took to achieve task goals), and subjective satisfaction (positive attitudes towards the use of the visualization) for reviewing Web search results.

\footnotetext{
${ }^{1}$ The project was funded by the European Commission under the Fourth Framework of the ESPRIT Program, Project No. 29232. www.insyder.com
} 
The evaluation results indicated some difficulties of user interaction with the system, e.g., more than $50 \%$ of the users voted for the ResultTable when asked with which visualization they performed best. Other visualizations were helpful as an addition to the ResultTable, but not as primary tools. When studying the expected value of the visualizations, it can be said that in the visualization (e.g. Scatterplot, BarGraph, SegmentView) plus ResultTable conditions where the user had the possibility to decide which component to use both components were used in the majority of cases. When analyzing usage times in these conditions, the ResultTable was the favorite component of the users. It was used in all three user interface test conditions with ScatterPlot, BarGraph, and SegmentView for more than $50 \%$ of the overall task time. Interpreting usage time as an indicator of expected value, the expected value of the ResultTable seemed to be higher than that of the other components for the users. Switching between completely different visualizations confused the users. Therefore, we tried to find a possibility to combine the regular table view with other views like the BarGraph or the SegmentView.

\section{The SuperTable + Scatterplot}

Based on the empirical findings of the evaluation we have decided to integrate the ResultTable, BarGraph, and SegmentView into one visualization called "SuperTable” and to improve the ScatterPlot.

In the user test the users requested a number of features for the BarGraph and the SegmentView already implemented in the ResultTable. All this could also be implemented in the ResultTable. Therefore, the proposed $\mathrm{Su}$ perTable integrates the concept of a distortion-based table, the BarGraph and the SegmentView (with TileBars and StackedColumns) in a way that allows easy manipulation of the table.

The redesign of the INSYDER visualizations combines the SuperTable + ScatterPlot into one single window offering different brushing techniques between them. Therefore the ScatterPlot will supplement the SuperTable by giving the user a quick overview of all search results, and offering the user a variety of controls (e.g. defining own views, zooming, selecting, filtering) to reduce the amount of hits to a smaller group of interesting documents. These documents can then be selected by the user and analyzed in more detail in the SuperTable.

The enhanced Scatterplot with additional lens mechanisms (e.g., a magic lens for filtering operations [4]), distortion techniques in both $\mathrm{X}$ - and $\mathrm{Y}$-dimensions (allowing a smooth transition between focus and context) and the radial MDP visualization tightly coupled with the SuperTable and a document browser (showing the detailed document with keyword highlighting) are our main redesign ideas.

\subsection{Discrete Change of Modalities: the Level Con- cept}

The first design variant of the SuperTable with the level concept, which is combined with the Scatterplot and a Browser window on a single panel is shown in Figure 1.

At first sight, the SuperTable doesn't differ from the original ResultTable: Every row represents the metadata of a web search result - in that case a html-page. The columns describe all characteristics concerning this document, e.g. title, url, date, size and so on. The main feature of the initial view is that no text at all will be seen in the table. There are just a number of multicolored bars representing numeric and textual data.

The distinction between the origin ResultTable and the SuperTable consists of four different views representing the "level of detail" for documents. We call this method of looking closer and closer at the details of a document the "Focus of Interest". The more you want to know about a document, the deeper you have to look, i.e. the higher the level has to be. It is possible to focus single documents, several documents or the whole list of documents.

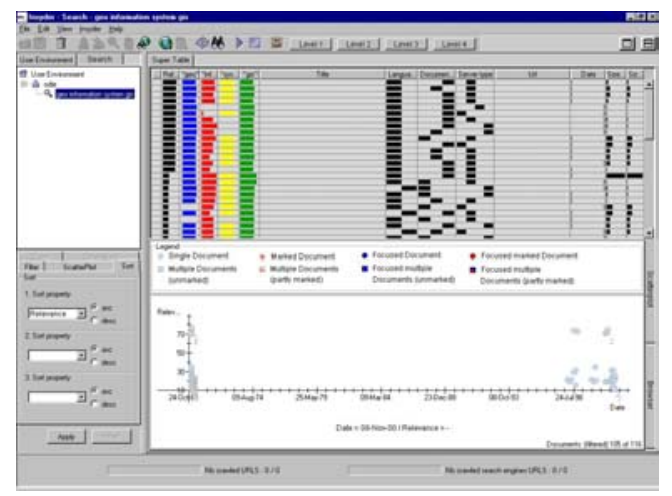

Figure 1: SuperTable Level 1

Level 1 represents an overview over all documents (Figure 1). All rows are as small as possible, so that in the best case all documents fit on the available space. Corresponding to the number of documents, the height of the rows can vary. Usually the rows will be too small to hold text, so only bars will be displayed. The length and the position of the bars encode various characteristics of the document depending on the type of data they represent. The length of the bars (representing numeric data like size, relevance) is equivalent to their numeric values. Nominal attributes can also be represented by a bar. For a few attributes we can code their values through position; for example, the language which may be English or French in our application (the left half of the cell means "English" and the right one means "French"). If there were too many different nominal values, visualization would be too confusing which is for example the case 
with the title. Therefore, this column will be empty. Different colors can additionally encode different search terms.

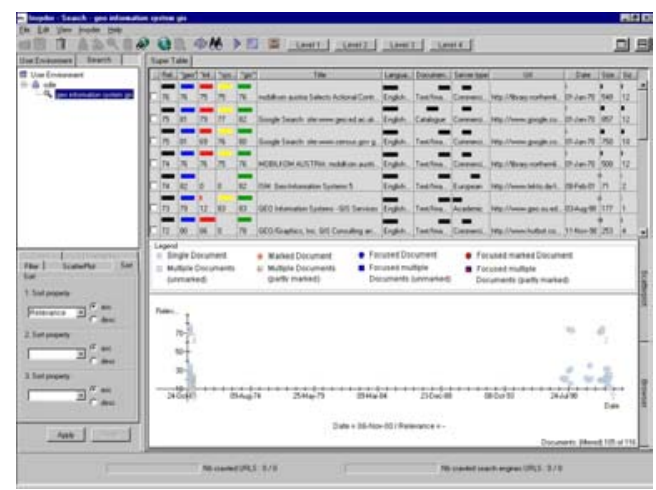

Figure 2: SuperTable Level 2

On Level 2 (Figure 2) more information will be visible in form of text completing the visual representation of the multicolored bars. Now numeric values add detailed information about the bar displays from the initial, graphics-only display. Title and URL are now readable, but only up to the width of the respective column. All wider texts become truncated, marked by three dots.

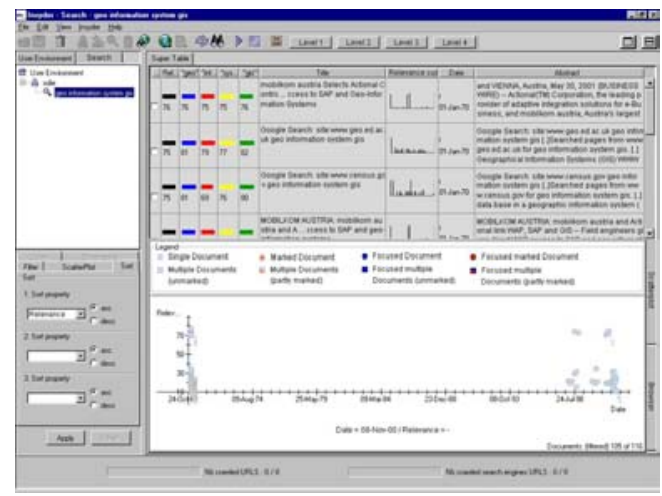

Figure 3: SuperTable Level 3

Level 3 provides the opportunity to read the whole text of those characteristics, which had to be abbreviated because of their size (Figure 3). Visualizations were cut off to gain space, so that title, URL and abstract are now completely visible. In addition a new column is introduced, the socalled "Relevance Curve". It represents a twodimensional chart of the whole document by dividing the document into a number of segments, e.g. sentences, subordinate clauses, etc. The height of single bars encodes the overall relevance for each individual segment.

Level 4 (Figure 4) displays only the most important values of a document. The title, an abstract and an extension of the relevance curve, the so-called Segment View which uses stacked columns or TileBars [6]. Which kind of query term distribution visualization will be used de- pends on the user preferences. In this case, every segment has the same length, not varying from one document to another. Consequentially the length of the SegmentView differs from document to document, always corresponding to the real length of the document. The stacked columns or TileBars are colored according to the colored search terms in the former levels. So it is easier to discover the segments where all search terms can be found, not just a few of them. You can spot segments which include only one or two search terms and discern these from parts including all terms. Some terms may be seen more important than others, so a segment with a lower relevance can be important although not all terms are included. Per default, instead of the Scatterplot a preview browser is offered showing the selected document or per default the first document of the SuperTable. This offers the user the greatest level of detail showing him the text of the document (without images) enhanced by keyword highlighting.

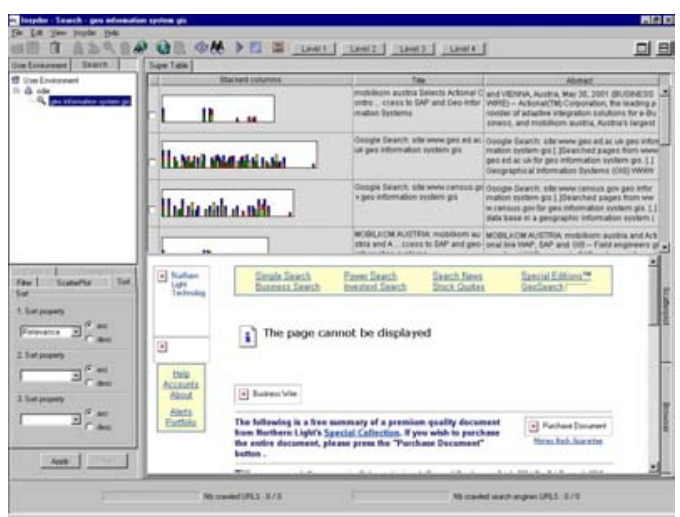

Figure 4: SuperTable Level 4

\subsection{Smooth Change of Modalities: the Granular- ity Concept}

Visualizations in document retrieval have one common flaw: the change of modalities during a search. The query is formulated in a textual way, for example by a simple list of search terms, a Boolean combination of search terms, a form, a SQL-statement and so on. Only a few systems allow a mere graphical input. In a next step a graphical version of the result set is presented. Here the user can see the effects of his query on the result set. Some visualization even allows manipulating the result set. Finally, the documents themselves are presented textually, usually in a selectable list of titles.

Thus, the user has to switch at least twice between modalities. The first time in order to get from his query formulation to the visualization and the second time in order to get from the visualization to the actual documents. Nevertheless, in practice, he will use iterative retrieval 
and therefore he will have to switch between the modalities much more often. Each time he has to connect the textual representation to the graphical mentally.

This cognitive load of connecting the modalities raises the question whether in this context visualization is sensible at all. In general, visualizations are employed because of the human visual capacities [2]. The human mind is able to cope with a comparably high amount of visual data and has serious problems of juggling with the same amount of textual data. Based on this fact, visualizations in document retrieval are mostly employed in order to explain the search result. Patterns and exceptions in the result set can easily be detected and the query reformulated appropriately.

However, this advantage of visualization is won at the cognitive expense of the transfer between the modalities. Here we want to introduce a possibility of reducing this expense. Systems like InfoCrystal [14] and DEViD [3] integrated the search into the visualization and thus reduced the problems of the first transfer. INSYDER might be evolved to solve the problem of the second transfer from the visualization to the textual output.

The level concept presented above tries to combine the visualizations employed by INSYDER in order to ease their interpretation. In a design variant, this level concept is further smoothened by introducing the concept of granularity. Granularity is a term used in photography to describe the accuracy of pictorial presentations on film. The higher the granularity, the more details can be seen on a picture.

This idea can be transferred to integrate the visualizations of the SuperTable smoothly: Using a very low granularity results in a simple histogram which states the overall importance of the single documents (Figure 5). A very high granularity would lead to a representation of the actual text (Figure 11). In between these two extreme granularities are as many intermediate steps as are required to give the impression of a smooth transition. Ideally, no distinct steps at all should be identifiable. However, in regard to the technical realization we would have to cope with steps. In order to emphasize the continuous transition the visualization is manipulated and adjusted by a slider comparable to the sliders used to adjust the volume of media players.

The granularity concept will be realized as seven different steps ranging from a simple 3-column view containing the relevance, an abstract text representation and the slider to a full text representation with the keywords highlighted. Between those steps there are five other visualizations giving the impression of "zooming into the document". Please note that the steps described in the following are not meant to be consecutive steps which the user has to follow during the retrieval process. They are meant to be alternative views which can be adjusted as a whole (like it is shown in this paper) or separately for each document.

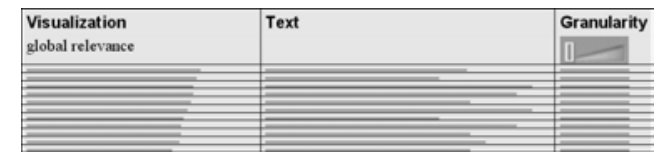

Figure 5: SuperTable, granularity step 1

Step 1 (Figure 5): The table consists of three columns: one for the visualization, one for the textual representation and one for the slider. The visualization is simple and just shows the global relevance. The text is not readable yet, because it is too small. The granularity slider is put on minimal granularity. Moving the slide bar further right maximizes the granularity: more details can be seen. There is a global slider for all documents and local sliders for each single document. The local sliders are nearly hidden because of the slimness of the rows. Moving the mouse over a row makes the sliders appear.

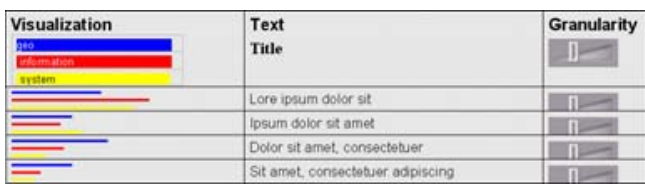

Figure 6: SuperTable, granularity step 2

Step 2 (Figure 6): The relevancies are shown in more detail: there is a color-coded bar for each search term. Ordering the color-coded terms vertically leads to a smooth transition from the global relevance to the single relevancies. The text column now contains the title of the document. The local sliders are visible.

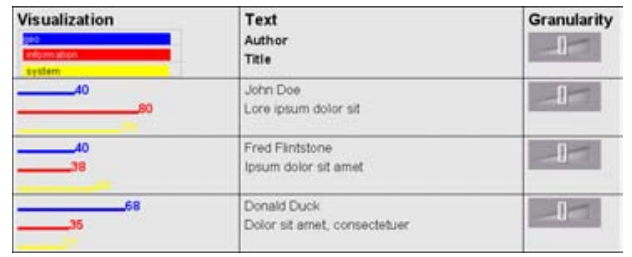

Figure 7: SuperTable, granularity step 3

Step 3 (Figure 7): Additionally, the numeric values of the relevancies will be shown next to the bar which increases the overall height of the row. This creates space in the text column to show additional data like author, size, language, and so on.

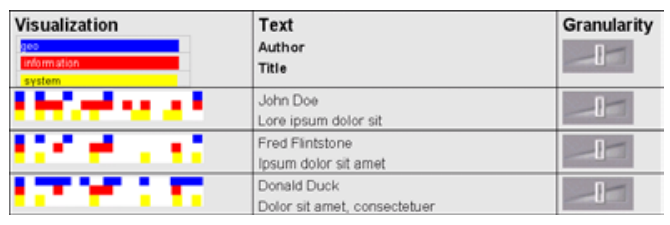

Figure 8: SuperTable, granularity step 4

Step 4 (Figure 8): Now the relevance bars are split up into TileBars. This means that the document is divided into segments, representing the columns of the TileBar. The 
occurrence of a query term in the respective segment is indicated by a colored tile. The color saturation indicates the frequency of occurrence of a query term.

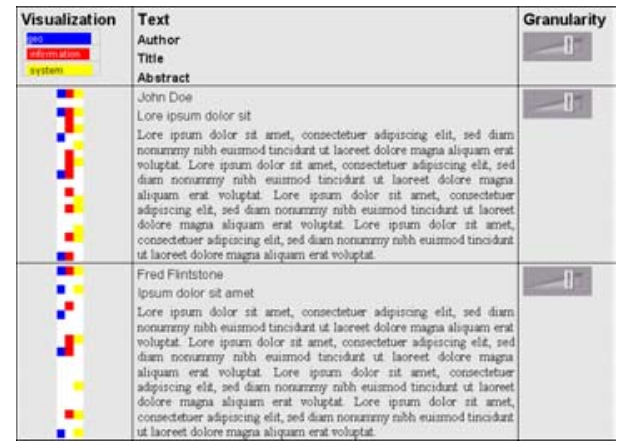

Figure 9: SuperTable, granularity step 5

Step 5 (Figure 9): This step is similar to the fourth step except that the TileBars are rotated by $90^{\circ}$. This creates additional space in the text column which can be used to show the abstract.

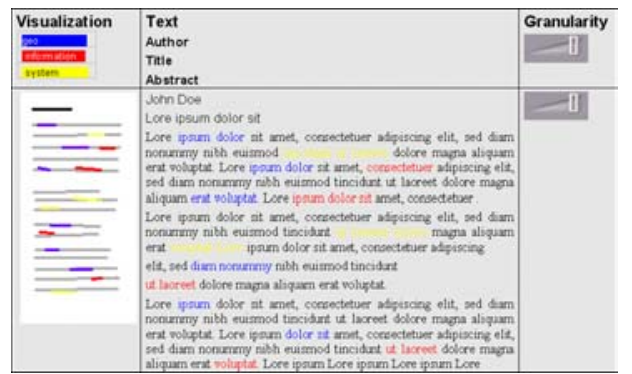

Figure 10: SuperTable, granularity step 6

Step 6 (Figure 10): Now the left column contains a thumbnail view of the document with the locations of the query terms highlighted. The second cell also uses color highlighting in the text. If possible, the abstract is replaced by the complete text.

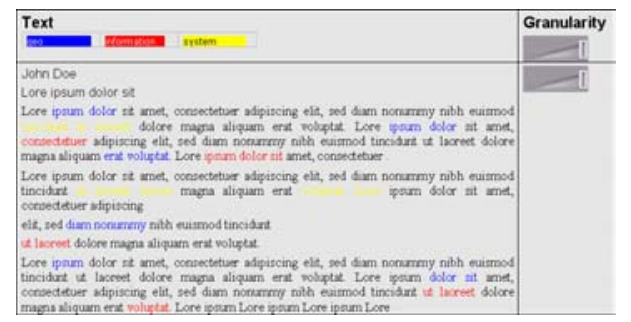

Figure 11: SuperTable, granularity step 7

Step 7 (Figure 11): The last step will be a one-column representation of the desired text with the query terms highlighted. Only two columns are left: the visualization column and the text column are merged with another. The maximal granularity (the whole text of the document) is reached. To retain the advantage of the visualization, the first two columns of step 6 are combined in a single one offering as much space as possible for the final text presentation.

Two goals can be achieved with this design variant of the SuperTable: First, the several visualizations do not appear as distinct anymore. On the contrary, the user has the feeling that he is working with one single visualization in different states. The interpretation should be heavily simplified and the users confusion of being confronted with different visualizations in the original INSYDER version will be eliminated.

Secondly, the SuperTable solves a problem which seemed to be immanent to visualizations in document retrieval: the change of modalities. During the retrieval process the user has to get past a modality change twice: one time from the textual input of the query to the visualization and a second time from the visualization to the textual output of the documents. These changes are usually rather harsh, because researchers focused too much on the visualizations themselves leaving aside their embedding in the retrieval process.

\section{Related Work}

The origin INSYDER system has been influenced by different existing systems. The use of the Scatterplot was mainly inspired by the visual information seeking systems Envision [7] and FilmFinder [1]. The use of the BarChart was mainly inspired by the work of [15] and the use of the TileBar by the work of [6].

The SuperTable idea has been influenced by distortionbased approaches using focus-plus-context techniques in a tabular data representation. The Table Lens [10] or FOCUS [13] are typical examples for this approach. Textual and graphical representations of the data are used in both systems. Focus-plus-context allows showing more cells of the data table on the screen then without this technique. In both systems, the coherence of rows and columns and their labels is preserved when distorting parts of the view. The graphical elements are used for pattern recognition when working with quantitative variables. Whereas in the Table Lens the cases are displayed in rows, in FOCUS they are displayed as columns.

\section{Conclusions and Outlook}

The advantage of the original INSYDER system was the variety of visualizations used to support users in their web search. Our redesign combines these visualizations with the widely adopted spread sheet-like layout in the SuperTable. So new possibilities are given to find the most appropriate document for the current task in an environment users are accustomed with. Both design approaches presented in this paper give us the possibility to 
improve the advantages of this combination in a more detailed way.

After the implementation of both versions is finished ${ }^{2}$, we will perform an evaluation (user tests) to compare these two redesign ideas and then we will decide, which approach will be preferable. Additionally, a highly sophisticated data model will enable us to adapt INSYDER to a wide range of application domains were metadata play an important role (e.g. digital libraries, web, geodata archives). Therefore, INSYDER should become a visualization framework for different application domains visualizing metadata.

We are currently adapting our visualization framework to a new application domain in a project called INVISIP (Information Visualization in Site Planning) ${ }^{3}$. The aim of this project is to support users in the retrieval process of complex application areas such as site planning and to facilitate graphic-interactive access to geodata archives. Here, the idea is to provide information visualization techniques in the different information retrieval phases to locate appropriate geodata, which is necessary to solve planning tasks, e.g. generation of ecological, environmental or socio-demographic reports. The SuperTable + Scatterplot will be introduced in a 3D GeoLibrary [5] as one new information visualization technique to support users during the different information retrieval phases, especially search result presentation and comparison of search results (hints for query modification).

\section{References}

[1] Ahlberg, Christopher; Shneiderman, Ben: Visual Information Seeking: Tight Coupling of Dynamic Query Filters with Starfield Displays. In: Adelson, B.; Dumais, S.; Olson, J. S. (Eds.): CHI 1994: Conference Proceedings Human Factors in Computing Systems. Conference: Boston, MA, April 24-28 1994. New York (ACM Press) 1994. p. 313-317.

[2] Card, Stuart; Mackinley, Jock; Shneiderman, Ben (Eds.) (1999). Readings in Information Visualization. Using Vision to Think. San Francisco.

[3] Eibl Maximilian: Visualisierung im Document Retrieval. Forschungsberichte Band 3, IZ Informationszentrum Sozialwissenschaften, Bonn, 2000

[4] Fishkin, Ken; Stone, Maureen C.: Enhanced Dynamic Queries via Movable Filters. In: Katz, Irvin R.; Mack, Robert L.; Marks, Linn et al. (Eds.): CHI 1995: Conference Proceedings Human Factors in Computing Systems. Conference: Denver, CO, May 7-11 1995. New York (ACM Press) 1995. p. 23-29.

${ }^{2}$ We are currently implementing the redesign variants in Java using the shown mockups (html- and paper-prototypes) as starting points.

${ }^{3}$ The project is funded by the European Commission under the Fifth Framework of the IST Program, Project No. IST-200029640, www.invisip.de
[5] Göbel, S., Haist, J., Goebel, C. GeoCrystal : GraphicInteractive Access to Geodata Archives. Proceedings SPIE 2002 - Visualization and Data Analysis, San Jose, CA, 2002.

[6] Hearst, Marti A.: TileBars: Visualization of Term Distribution Information in Full Text Information Access. In: Katz, Irvin R.; Mack, Robert L.; Marks, Linn et al. (Eds.): $C H I$ 1995: Conference Proceedings Human Factors in Computing Systems. Conference: Denver, CO, May 7-11 1995. New York (ACM Press) 1995. p. 59-66.

[7] Nowell, Lucy T.; France, Robert K.; Hix, Deborah et al.: Visualizing Search Results: Some Alternatives to QueryDocument Similarity. In: Frei, Hans-Peter; Harman, Donna K.; Schäuble, Peter et al. (Eds.): SIGIR 1996: Proceedings of the 19th Annual International ACM SIGIR Conference on Research and Development in Information Retrieval. Conference: Zürich, Switzerland, August 18 -22 1996. New York (ACM Press) 1996. p. 67-75.

[8] Mann, T.H., Reiterer H.: A Combined Visualization Approach for $W W W$-Search Results in: Proceedings of the IEEE Visualization 1999 (Vis '99), San Francisco

[9] Mann, Thomas M: Visualization of Search Results from the World Wide Web, University of Konstanz, 2002, http://www.ub.uni-konstanz.de/kops/volltexte/2002/751/

[10] Rao, Ramana; Card, Stuart K.: The Table Lens. Merging graphical and symbolic representations in an interactive focus + context visualization for tabular information. In: Adelson, B.; Dumais, S.; Olson, J. S. (Eds.): CHI 1994: Conference Proceedings Human Factors in Computing Systems. Conference: Boston, MA, April 24-28 1994. New York (ACM Press) 1994. p. 318-322.

[11] Reiterer, Harald; Mußler, Gabriela; Mann, Thomas; Handschuh, Siegfried: INSYDER - An Information Assistant for Business Intelligence, Proceedings of the 23 Annual International ACM SIGIR 2000 Conference on Research and Development in Information Retrieval, ACM press, 2000, pp.112-119

[12] Reiterer, Harald; Mußler, Gabriela; Mann, Thomas M.: Visual Information Retrieval for the $W W W$, in: Smith M.J. et al. (eds.), Proc. HCI International 2001, New Orleans, Lawrence Erlbaum, 2001, pp. 1150-1154

[13] Spenke, Michael; Beilken, Christian; Berlage, Thomas: FOCUS: The Interactive Table for Product Comparison and Selection. In: UIST 96: 9th ACM Symposium on User Interface Software and Technology New York (ACM Press) 1996. p. 41-50.

[14] Spoerri, Anselm (1994a). InfoCrystal: Integrating Exact and Partial Matching Approaches through Visualization. In: RIAO'94 Proceedings, Oct.11-13, 1994 New York (NY), S.687-696.

[15] Veerasamy, Aravindan; Navathe, Shamkant B.: Querying, Navigating and Visualizing a Digital Library Catalog. In: Digital Libraries 1995: The Second Annual Conference on the Theory and Practice of Digital Libraries. Conference: Austin, TX, June 11-13 1995. 